bord terminal aussi large, mais un peu plus oblique que chez vidua. Thorax large de כ̋,כ̆ mill., mésonotum long de 6 mill., avec un sillon médian qui le traverse presque entièrement. Gastre plus large en avant, un peu rétréci en arrière ( ${ }^{\mathrm{er}}$ segment large de 8,כ̆ mill., $2^{\mathrm{e}} \mathrm{de}$ $8,2,3^{e}$ de $7, \breve{s}$ mill.). Les ailes manquent. Du reste semblable à vidua $\mathrm{Sm}$. Diffère à première vue de C. vidua var. Junodi For. par la disposition inverse des couleurs.

Afrique orientale anglaise (Ch. Alluaud). Reçu du Muséum de Paris.

\title{
Description de trois nouvelles espèces de Lépidoptères hétérocères de Cochinchine
}

par J. DE JoAnnis.

II. le commandant Fouguet a eu l'obligeance de m'envoyer un certain nombre d'espèces intéressantes de Lépidoptères de Cochinchine, parmi lesquelles je décris ici deux Syntomidae et une Noctuidae.

1. Geryx Fouqueti, n. sp. - Exp. al. : 22 mill. - Fronte alba, capite nigro, antennis pectinatis nigris, collari et thorace nigris, siapulis albis ad basim, deinde nigris; abdomine cum sex primis annulis supra et infra rubro-roseis, tertio et quarto supra et infra et quinto infra tantum leviter nigro marginatis; ultimis annulis nigro-cyaneis purpurascentibus. Pedibus nigris, femoribus omnibus interne albis. Alis anticis nigro-purpureis, lata macula hyalina subtriangulari prope basim infra cellulam, secunda triangulari intra cellulam, tertia subrectangulari post cellulam a vena 4 divisa, tandem quarta elongata inter venas 6 et $\%$; posticis hyalinis nigro-purpureo marginatis, latius ad apicem.

Front blanc, tête noire, les yeux bordés de blanc en arrière, palpes courts, noirs, frangés de longs poils en dessous; antennes noires (la pointe est brisée), très pectinées; collier et thorax noirs, ptérygodes blancs à la base, noirs à la pointe. Abdomen avec les six premiers segments d'un rouge carmin foncé, les $3^{\text {e }}$ et $4^{\mathrm{e}}$ anneaux seuls bordés d'un fin liséré brun noir; les derniers segments noir bleu à reflets pourpres. Pattes noires avec les cuisses blanches en dedans aux trois paires. Poitrine brune avec quelques reflets cuivrés. Abdomen 
en dessous comme en dessus, mais le כॅe anneau est aussi liséré de brun noir. Ailes supérieures noir pourpre, avec une grande tache subtriangulaire hyaline au-dessous de la cellule, surmontée d'une tache triangulaire semblable dans la cellule; une autre tache hyaline ovale subrectangulaire au delà de la cellule coupée par la nervure 4, audessus de laquelle s'en trouve une quatrième, allongée, entre les nervures 6 et 7 .

Un $\sigma^{\top}$ provenant du cap St-Jacques (Cochinchine), pris en septembre 1910. Je dédie cette belle espèce au commandant Fouquet. Elle se placerait près de $C$. flaviplagia Hmpsn.

2. Eressa rubribasis, n. sp. - Exp. al. : 23 mill. - Fronte alba, capite nigro; antennis crenulatis, nigris, ultima parte alba; scapulis albis ad basim, deinde nigris. Thorace nigro, abdomine nigro-cyanescenti, duobus primis annulis rubro-roseis, corpore infra brunneo, pectore ad latera rubro-roseo. Coxis posteriorum pedum albis. Alis hyalinis exceptis, in anticis, apice late nigro, intervallo inter venas 5 et 6 et parte distali intervalli inter 2 et 3, quae usque ad angulum externum macula nigro brunnea prolongatur; in posticis, margine tenui, paulo latiore ad apicem.

Front blanc, tête noire, quelques écailles blanches en arrière autour de l'œil, palpes courts squameux. Antennes noires sauf la pointe qui est blanche, très fortement crénelées, presque pectinées; ptérygodes blancs à la base, noirs à la pointe. Thorax noir; abdomen noir avec quelques reflets bleus métalliques, les deux premiers segments rouge carmin. Dessous brun, les côtés de la poitrine rouge carmin, et les hanches de la troisième paire de pattes blanches. Ailes presque complètement hyalines sauf, aux antérieures, une portion assez large à l'apex, l'intervalle des nervures 5 et 6 entier et la moitié distale de l'intervalle entre 2 et 3 qui est prolongée par la teinte noire jusqu'à l'angle externe. Aux ailes postérieures un simple liséré noir très mince, un peu plus marqué dans la région apicale.

Un $\sigma^{\top}$ provenant de Thudaumot (Cochinchine) et pris en décembre 1911.

Cette espèce me semble pouvoir se placer près de $E$. annosa Wlk.

3. Cerocala orientalis, n. sp. - - Exp. al. : 37 mill. - Anticis nigro-brunneis, antemediana tenui, nigra, recta, parum obliqua; macula orbiculari nigra, reniformi brunneo-nigra, intus nigro marginata; postmediana tenui, nigra, recta a costa ad venam 3 , inde valde recurva 
usque ad maculam reniformem, dein leviter arcuata usque ad marginem internum; regione media albescenti, squamis nigro-brunnneis conspersa; squamis metallescentibus in basi, lineam sinuatam tenuem efformantibus, item in maculis orbiculari et reniformi, et post lineam post. medianam; submarginali albida, denticulata, nigro-brunneo marginata; costa et margine externo griseis squamis conspersis; linea marginali nigra; ciliis brunneo-nigris. Posticis brunneo-nigris, regione externa saturatiore; lunula discali nigra, cum fascia alba illam consequente versus marginem internum obsolescente; linea submarginali albida, valde obsoleta praeter ad angulum analem; ciliis albis ab apice ad venam 4, a vena 2 ad venam 1 a et ad marginem internum.

Infra alis albis praeter marginem externum late nigrum, cum subterminali albida, incompleta, item et lunulam discalem nigram, in anticis cum fascia obliqua ipsam continuante usque ad angulum externum et in posticis cum maculis nigrescentibus, interruptis et obsoletis, fasciam transversam formantibus. Ciliis ut supra.

Capite, thorace et abdomine brunneo-griseis; collari albescente; palpis albis, tertio articulo brunnescente; corpore subtus albido, pedibus squamis brunneis leviter conspersis. Antennis filiformibus, albidis.

Les ailes supérieures ont la région basilaire brun noir un peu éclairée vers la base, près du bord interne; elle est limitée par une ligne antémédiane fine, noire, presque complètement droite, un peu oblique, à peine un peu convexe près du bord interne, précédée d'une ligne d'écailles plombées, sinueuse, fine, interrompue. La région discale a le fondblanc, fortement saupoudrée d'écailles noirâtres; la tache orbiculaire noire avec, au centre, un paquet en relief d'écailles plombées; tache réniforme assez large limitée intérieurement par un bourrelet noir recouvert d'écailles plombées et parcourue en haut et en bas de deux traînées longitudinales d'écailles semblables. Ligne postmédiane très droite et parallèle à l'antémédiane de la côte à la nervure 3 , se recourbant légèrement ensuite en arrière et allant toucher la subterminale, puis rentrant vivement et formant la sinuosité ordinaire, puis venant toucher le bas de la réniforme, puis se dirìgeant vers le bord interne en formant une légère convèxité du còté de la base. Cette ligne est fine et noire et elle borde un espace brun noir qui s'étend jusqu'à la subterminale; dans cet espace se trouve une ligne plombée, assez large, commençant un peu au-dessous de la côte et finissant à la nervure 3, puis une ligne arquée semblable à l’intérieur du sinus formé par la partie inférieure de la postmédiane; subterminale blanchâtre, fine, dentée sur les nervures 7, כ̆ et sur le pli dorsal; elle est bordée extérieurement par une ombre brun noir commençant juste à l'apex et se continuant 
absolument droite sauf une dent sur la nervure כ̆. La région marginale est grise, un peu saupoudrée de noir près du bord externe. La côte est également teintée de gris jusqu'à la postmédiane et on voit deux traits costaux blanchâtres avant la subterminale. Une ligne marginale fine brun noir. Frange brunâtre, un peu plus claire à la pointe.

Ailes inférieures brun noirâtre, un peu plus pâles dans la région basilaire qui s’étend jusqu'à la lunule discoïdale forte et noire et suivie d'une bande discale d'un blanc pur de la côte à la nervure 2, continuée dans la région abdominale par une étroite trainée blanchâtre; bande subterminale d'écailles blanchâtres très obsolète, bien nette seulement près de l'angle anal. Frange blanche au bord interne, entre $\mathbf{1}$ a et $\mathbf{2}$, puis de $\mathbf{4}$ au sommet de l'aile, le reste noirâtre.

Dessous blanc pur avec une large bordure noire aux quatre ailes, traversée par une bande blanche subterminale incomplète et interrompue; une forte lunule noire, prolongée aux supérieures par une trainée noirâtre venant à l'angle interne, et aux inférieures précédée d'une petite tache costale noire et suivie de quelques taches brunâtres abdominales, de façon à former une sorte de bande transversale très discontinue. Frange aux inférieures noirâtre sauf une petite portion blanche à l'apex et une petite éclaircie entre les nervures 1 et 3 ; aux inférieures comme en dessus.

Tète et thorax gris, collier brun noir précédé d'une collerette blanchàtre; abdomen gris plus clair. Palpes blancs, le troisième article lavè de brunâtre. Dessous du corps blanc, pattes blanchâtres avec quelques écailles brunes. Antennes filiformes, blanchâtres.

Un exemplaire $q$.

M. le commandant Fouguet a pris cette remarquable espèce au cap St-Jacques (Cochinchine) en octobre 1910. Il m'a fait savoir qu'en mème temps que cette $\mathcal{q}_{\text {, }}$ il avait pris un exemplaire $\sigma^{\Upsilon}$ à longues antennes pectinées.

C'est le premier exemple d'une espèce de Cerocala provenant des régions orientales. Ce genre n'était encore connu que d'Europe méridionale, d'Afrique et des régions occidentales de l'Asie (Syrie, etc.). Le point le plus oriental d'où ce genre avait été signalé parait ètre Socotora. De plus, les Cerocala habitent en général les régions sèches et désertiques. La nouvelle localité ne peut rentrer à aucun titre sous une telle rubrique, le cap $\mathrm{S}^{\mathrm{t}}$-Jacques renfermant surtout des forêts et des marécages. 

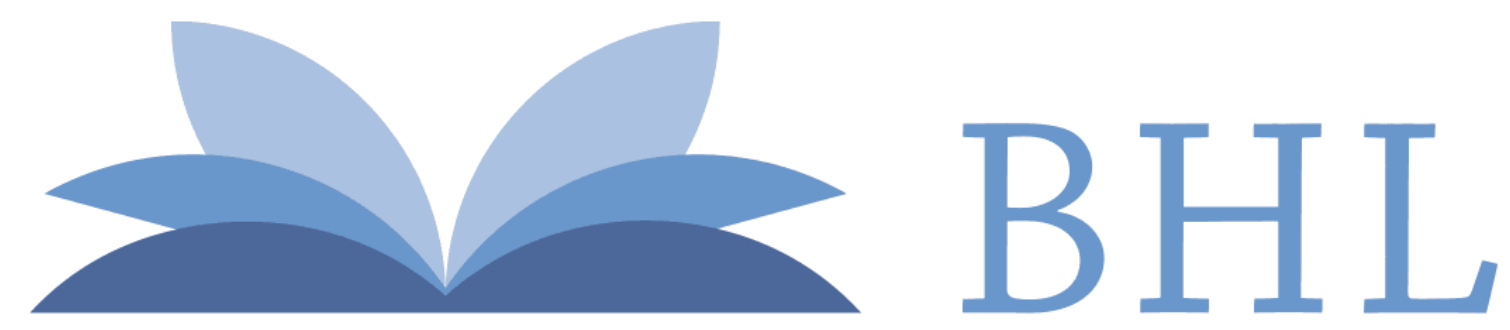

\section{Biodiversity Heritage Library}

1912. "Description de trois nouvelles especes de Lepidopteres heteroceres de Cochinchine." Bulletin de la Société entomologique de France 1912, 286-289.

View This Item Online: https://www.biodiversitylibrary.org/item/36402

Permalink: https://www.biodiversitylibrary.org/partpdf/40312

\section{Holding Institution}

Smithsonian Libraries

\section{Sponsored by}

Smithsonian

\section{Copyright \& Reuse}

Copyright Status: NOT_IN_COPYRIGHT

This document was created from content at the Biodiversity Heritage Library, the world's largest open access digital library for biodiversity literature and archives. Visit BHL at https://www.biodiversitylibrary.org. 